\title{
Characterization of Two Cryptic Plasmids Isolated in Haiti from Clinical Vibrio cholerae Non-01/Non-0139
}

\author{
Daniela Ceccarelli ${ }^{1,2 * t}$, Geneviève Garriss ${ }^{3 \dagger}$, Seon Y. Choi ${ }^{1,4}$, Nur A. Hasan 4,5 , \\ Ramunas Stepanauskas ${ }^{6}$, Mihai Pop ${ }^{5}$, Anwar Huq ${ }^{1,7}$ and Rita R. Colwell ${ }^{1,4,5,8 *}$
}

1 Department of Cell Biology and Molecular Genetics, Maryland Pathogen Research Institute, University of Maryland, College Park, MD, United States, ${ }^{2}$ Department of Bacteriology and Epidemiology, Wageningen Bioveterinary Research, Lelystad, Netherlands, ${ }^{3}$ Department of Microbiology, Cell Biology and Tumor Biology, Karolinska Institutet, Stockholm, Sweden, ${ }^{4}$ CosmosID Inc., Rockville, MD, United States, ${ }^{5}$ University of Maryland Institute for Advanced Computer Studies, University of Maryland, College Park, MD, United States, ${ }^{6}$ Bigelow Laboratory for Ocean Sciences, East Boothbay, ME, United States, ${ }^{7}$ Maryland Institute of Applied Environmental Health, University of Maryland, College Park, MD, United States, ${ }^{8}$ Department of Environmental Health Sciences, Bloomberg School of Public Health, Johns Hopkins University, Baltimore, MD, United States

Edited by: Feng Gao,

Tianjin University, China

Reviewed by: Elisabeth Margaretha Bik, uBiome (United States), United States M. Jahangir Alam, University of Houston, United States Eckhard Strauch Federal Institute for Risk Assessment, Germany

*Correspondence: Daniela Ceccarelli daniela.ceccarelli@wur.nl Rita R. Colwell

rcolwell@umiacs.umd.edu

${ }^{\dagger}$ These authors have contributed equally to this work

Specialty section:

This article was submitted to Evolutionary and Genomic Microbiology,

a section of the journal

Frontiers in Microbiology

Received: 30 August 2017 Accepted: 06 November 2017 Published: 23 November 2017

Citation:

Ceccarelli D, Garriss G, Choi SY, Hasan NA, Stepanauskas R, Pop M,

Huq A and Colwell RR (2017)

Characterization of Two Cryptic

Plasmids Isolated in Haiti from Clinical Vibrio cholerae Non-01/Non-0139.

Front. Microbiol. 8:2283.

doi: 10.3389/fmicb.2017.02283
We report the complete sequence of two novel plasmids, $\mathrm{pSDH}-1$ and $\mathrm{pSDH}-2$, isolated from clinical Vibrio cholerae non-O1/non-0139 during the early phase of the 2010 Haitian cholera epidemic. Plasmids were revealed by employing single-cell genomics and their genome content suggests self-mobilization and, for $\mathrm{pSDH}-2$, a toxin-antitoxin (TA) system for plasmid stabilization was identified. The putative origin of replication of pSDH-2 was mapped suggesting it replicates following the ColE1 model of plasmid replication. $\mathrm{pSDH}-1$ and $\mathrm{pSDH}-2$ were widespread among environmental $V$. cholerae non-O1/non-O139 with variable prevalence in four Haitian Departments. pSDH-2 was the most common element, either alone or with $\mathrm{pSDH}-1$. The two plasmids detection adds to the composite scenario of mobile genetic elements (MGEs) observed in $V$. cholerae in Haiti. The role these small cryptic plasmids circulating in Vibrio spp. play in bacterial fitness or pathogenicity merits further investigation.

Keywords: Vibrio cholerae, non-01/non-0139, Haiti, plasmid, cholera

\section{INTRODUCTION}

In January 2010, Haiti was struck by a devastating earthquake responsible of a humanitarian crisis still ongoing. Since the first case was diagnosed in October 2010 (World Health Organization, 2010) cholera remains a serious health threat in Haiti. 41,421 new cases were reported by the WHO for Haiti alone in 2016 (World Health Organization, 2017), a reminder of how significant the epidemic still is. The agent of epidemic cholera was recognized as Vibrio cholerae toxigenic serogroup O1 (Ceccarelli et al., 2011; Chin et al., 2011) although a population of V. cholerae nonO1/non-O139 was shown to coexist with clinical $V$. cholerae $\mathrm{O} 1$ in Haiti early in the epidemic (Hasan et al., 2012). V. cholerae non-O1/non-O139 have been isolated in Haiti, mostly from the aquatic environment (Baron et al., 2016), but proof of their presence in stools collected from asymptomatic Haitian infants two years prior to the earthquake has been published (Liu et al., 2014). $V$. cholerae non-O1/non-O139 are natural inhabitants of estuarine and coastal waters and, as opportunistic pathogens, can be responsible for infections other than cholera, generally through 
the consumption of raw or undercooked seafood. Non-O1/nonO139 V. cholerae infections are continuously reported worldwide (Chomvarin et al., 2014; Crowe et al., 2016; Hirk et al., 2016), emphasizing their growing clinical significance.

Mobile genetic elements (MGEs) play an essential role in gene transfer. The high incidence of plasmids in marine Vibrios (Hazen et al., 2007; Zhang et al., 2012; Aedo et al., 2014; Wang et al., 2016) suggests that the marine environment is an important source of genome plasticity mediated by acquisition of MGEs and recombination. The Haitian environment is no exception and $V$. cholerae non-O1/non-O139 has shown a higher genomic variability compared to clinical $V$. cholerae $\mathrm{O} 1$. We previously reported the presence of the SXT/R391-related integrating conjugative element ICEVchHai2 circulating among closely related $V$. cholerae non-O1/non-O139 (Ceccarelli et al., 2013), carrying new genes involved in recombination. More recently, we identified MGIVchHai6, a novel mobilizable genomic island (MGI) containing a mercury resistance transposon and an integron conferring resistance to chloramphenicol, trimethoprim and streptomycin/spectinomycin (Carraro et al., 2016). MGIVchHai6, whose sibling MGIs have also been detected in silico in the Indian subcontinent, North and South America, can be mobilized by IncA/C plasmids, highly conjugative elements also known to circulate in Haitian V. cholerae (Folster et al., 2014).

While performing genomic heterogeneity tests on Haitian clinical strain $V$. cholerae $\mathrm{HC}-1 \mathrm{~A} 2$, using single cell genomics techniques (Stepanauskas, 2012), we unexpectedly recovered two novel plasmids. Complete sequence and distribution of these plasmids in clinical and environmental $V$. cholerae non-O1/non$\mathrm{O} 139$ and $\mathrm{O} 1$ isolated during the early days of the 2010 epidemic in Haiti are here reported, adding up to the composite scenario of MGEs circulating in Haitian V. cholerae.

\section{MATERIALS AND METHODS}

\section{Plasmid Sequencing and Annotation}

$V$. cholerae non-O1/non-O139 strain HC-1A2 was isolated from stool sample of a cholera patient in Saint-Marc (Artibonite) in 2010, with traditional methods of isolation and identification as previously described (Hasan et al., 2012). 317 single amplified genomes (SAGs) of $V$. cholerae $\mathrm{HC}-1 \mathrm{~A} 2$ were generated at the Bigelow Laboratory Single Cell Genomics Center (scgc.bigelow.org), as previously described (Swan et al., 2013). Briefly, single cell DNA genome sequencing involves isolating a single cell and performing whole-genome-amplification; this step is then followed by construction of sequencing libraries and DNA sequencing by a next-generation sequencer. One of the SAGs of $V$. cholerae HC-1A2, AD-538-E13, was genomically sequenced as follows. Single cell multiple displacement amplification products were sheared with M220 Focused Ultrasonicator (Covaris, Woburn, MA, USA) and 450-550 bp fragments were size-selected with BluePippin (Sage Science, Beverly, MA, USA). Paired-end sequencing libraries were generated using NEBNext Ultra DNA Library Prep kit (New England Biolabs, Ipswich, MA, USA), and 14 million $2 \times 250$ bp reads were produced using MiSeq (Illumina, San Diego, CA). The obtained sequence reads were quality-trimmed with Trimmomatic v0.32 5 using the following settings: -phred33 LEADING:0 TRAILING:5 SLIDINGWINDOW:4:15 MINLEN:36. Human DNA ( $\geq 95 \%$ identity to $H$. sapiens reference assembly GRCh38) and low complexity reads (containing $<5 \%$ of any nucleotide) were removed. The quality-filtered reads were digitally normalized with kmernorm 1.05 (http://sourceforge.net/ projects/kmernorm) using settings -k 18 -t 80 -c 2 and de novo assembled with Geneious 7.1.2 (Biomatters, Auckland, New Zealand) using medium-low sensitivity and allowing for circularization. Circular contigs with no homology to the previously sequenced $V$. cholerae HC-1A2 genome (Hasan et al., 2012) were selected for further analysis. The annotation of these putative plasmids was performed using RAST and GLIMMER. RAST subsystem for functional annotation was used to determine position 1 of the assembled plasmids. Database searches were carried out through NCBI using ORF Finder, BLASTn, BLASTp, and SMART (Schultz et al., 1998; Letunic et al., 2015).

\section{Bacterial Isolates}

By the end of October 2010 cholera had been confirmed in four of Haiti's 10 departments (administrative regions): Artibonite, Centre, Nord and Ouest, including the capital Port-au-Prince, and the capital's Cité Soleil district. In November 2010 sampling in these four Haitian departments took place; sampling details and isolation procedures are described elsewhere (Hasan et al., 2012). 175 Haitian bacterial strains isolated during this sampling campaign were screened for presence of plasmids pSDH-1 and pSDH-2: 70 clinical $V$. cholerae O1, 50 clinical $V$. cholerae nonO1/non-O139, 22 environmental $V$. cholerae non-O1/non-O139 and 33 isolates of Vibrio spp. and Aeromonas spp. Antiserum kits for V. cholerae O1 (V. cholerae Antiserum Poly; Difco, USA) and $V$. cholerae O139 (O139 Bengal; Hardy Diagnostics, USA) were used to determine serotype by slide agglutination, according to manufacturers' instructions. Serotyping was confirmed by multiplex PCR (Hoshino et al., 1998). Bacterial isolates were stored at $-80^{\circ} \mathrm{C}$ in LB broth containing $50 \%$ (vol $/ \mathrm{vol}$ ) glycerol.

\section{DNA Extraction and PCR Amplification}

Genomic DNA was extracted by boiling method as previously described (Ausubel et al., 1990). PCR assays were performed employing two sets of primers per plasmid designed using Primer3 (http://www.simgene.com/Primer3): set 1 (p1_cds1-Fw: AACAGATGGCGCAATCATAA; p1_cds5- Rev: CGCTCGTCA ATCGTCCTATG) and set2 (p1_inter-Fw: TGAATTAAGCCCG TTGGACT; p1_cds7-Rev: CAATTAAACATCCTGACTTTGA AAAA) for pSDH-1; set3 (p2_inter-Fw: ACAAGGCTAGCCCA CCTGTA; p2_cds1-Rev: ATTAGCAGCTGCGCGATTAG) and set4 (p2_cds4-Fw: GGTTGGGTCATTTGTTCCAT; p2_cds6Rev: AGTGTCCCTCATCGAAATGC) for pSDH-2. Amplicon sizes were $1,671,1,112,1,110$, and 1,495 bp, respectively. Thermal cycling conditions were as follows: (i) $5 \mathrm{~min}$ at $95^{\circ} \mathrm{C}$; (ii) 35 cycles of $30 \mathrm{~s}$ at $95^{\circ} \mathrm{C}, 30 \mathrm{~s}$ at $64^{\circ} \mathrm{C}$, and $2 \mathrm{~min}$ at $72^{\circ} \mathrm{C}$; and (iii) $5 \mathrm{~min}$ at $72^{\circ} \mathrm{C}$. PCR was performed in a $25-\mu 1$ reaction mix containing $12.5 \mu \mathrm{l}$ of GoTaq master mix polymerase (Promega). PCR amplicons were purified using a QIAquick PCR purification kit (Qiagen) according to manufacturer's instructions, and DNA 
sequences were determined by Eurofins Genomics (Huntsville, AL, USA).

\section{Nucleotide Sequence Accession Numbers}

Plasmid sequences have been submitted to the GenBank database with accession numbers: KY486775 (pSDH-1) and KY486774 (pSDH-2).

\section{RESULTS AND DISCUSSION}

Single-cell genomics performed on the pure culture of strain $V$. cholerae HC-1A2 with the aim of performing genomic heterogeneity tests revealed the presence of two previously unknown, circular plasmids pSDH-1 (4,985 bp) and pSDH-2 (5,580 bp) (Figure 1). We hypothesize that these two plasmids were likely missed during the original sequencing of $V$. cholerae isolates (Hasan et al., 2012) due to the shotgun library construction step (with $\sim 3 \mathrm{~kb}$ insert).

\section{Identification and Genomic Structure of Plasmid pSDH-1}

We found that plasmid pSDH-1 encodes 10 putative open reading frames (ORFs) (Figure 1 and Table 1). orf1 to orf4 and orf6 encode putative proteins of unknown function for which no known homologs could be found. BlastP search revealed that the predicted protein encoded by orf5 shows $45 \%$ identity to a replicase family protein from plasmid pRGRH0347, and contains replicase (RepA) and priCT_1 (primase C-terminal) domains. It is possible that this protein is involved in replication of $\mathrm{pSDH}-1$. Accordingly, orf5 was renamed repA.

orf7 and orf8 were respectively renamed mobA and mobC. The protein encoded by mobA shares homology with a relaxase protein from Vibrio parahaemolyticus, although similarity was observed for only part of the protein sequence. The product of mobC shares low similarity (33\%) with MobC from pMOMA1, a small cryptic plasmid recently described in Moraxella macacae (Whitehouse et al., 2015), but has no conserved domain hits. In various instances (Francia et al., 2004) MobA/MobC proteins have been shown to be part of a relaxosome, a protein complex required for initiation of transfer of conjugative and mobilizable elements. Conjugative elements encode all the functions required for their own transfer from a donor to a recipient cell, such as the proteins encoding the conjugative machinery and the components of the relaxosome. On the other hand, mobilizable elements rely on the conjugative machinery provided by a coresiding conjugative element. In most cases mobilizable elements encode their own relaxase, but some cases of trans-acting relaxases have been reported (Daccord et al., 2010; Carraro et al., 2017). The putative MobA protein encoded by pSDH-1 seems to be related to the $\mathrm{MOB}_{\mathrm{HEN}}$ family of relaxases (Francia et al., 2004), to which the ColE1 relaxase belongs. Although, it shares only low overall similarity with the relaxases of this family, the conserved motifs previously described are perfectly conserved in pSDH-1 MobA, with the exception of proline in motif III. The $\mathrm{MOB}_{\mathrm{HEN}}$ family relaxases are typically associated with accessory proteins, namely MobC, normally encoded by a
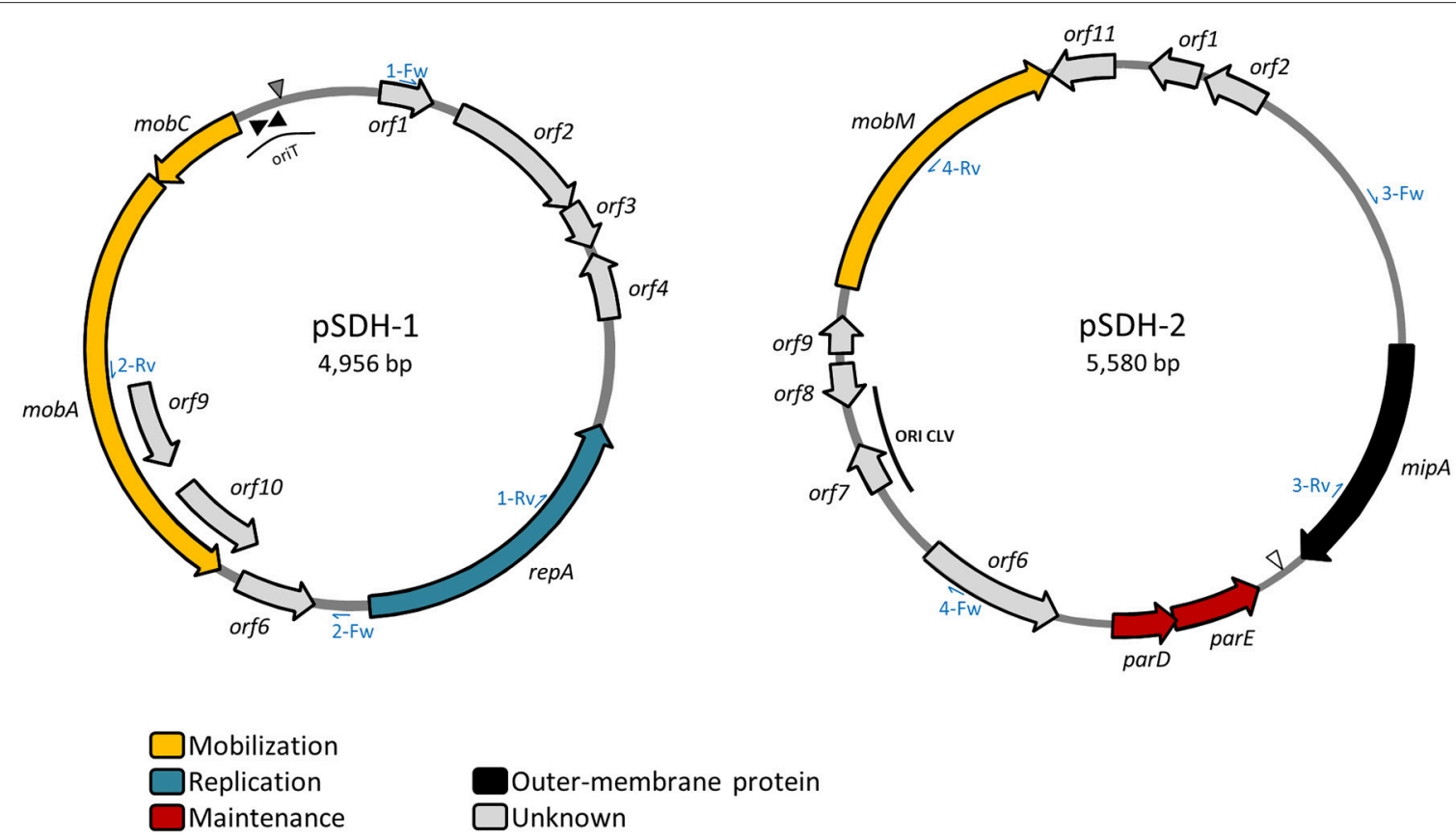

Outer-membrane protein Unknown

FIGURE 1 | Schematic representation of plasmids pSDH-1 and pSDH-2. Predicted open reading frames (ORFs) are indicated by arrows pointing in the direction of transcription. ORFs to which a putative function could be assigned are color-coded. Size of the plasmids is indicated. Position of the inverted repeats of the putative oriT region of $\mathrm{pSDH}-1$ are indicated by black triangles. Location of the putative nic site is indicated by a gray triangle. The open triangle indicates the position of the putative xer recombination site of pSDH-2. The position of the putative CLV-like origin of replication of pSDH-2 is indicated. Position of PCR primers used for screening for the presence of both plasmids are indicated by blue half-arrows and are labeled with the number of the set followed by Fw (forward) or Rv (reverse). 
TABLE 1 | ORF content of $\mathrm{pSDH}-1$ and $\mathrm{pSDH}-2$.

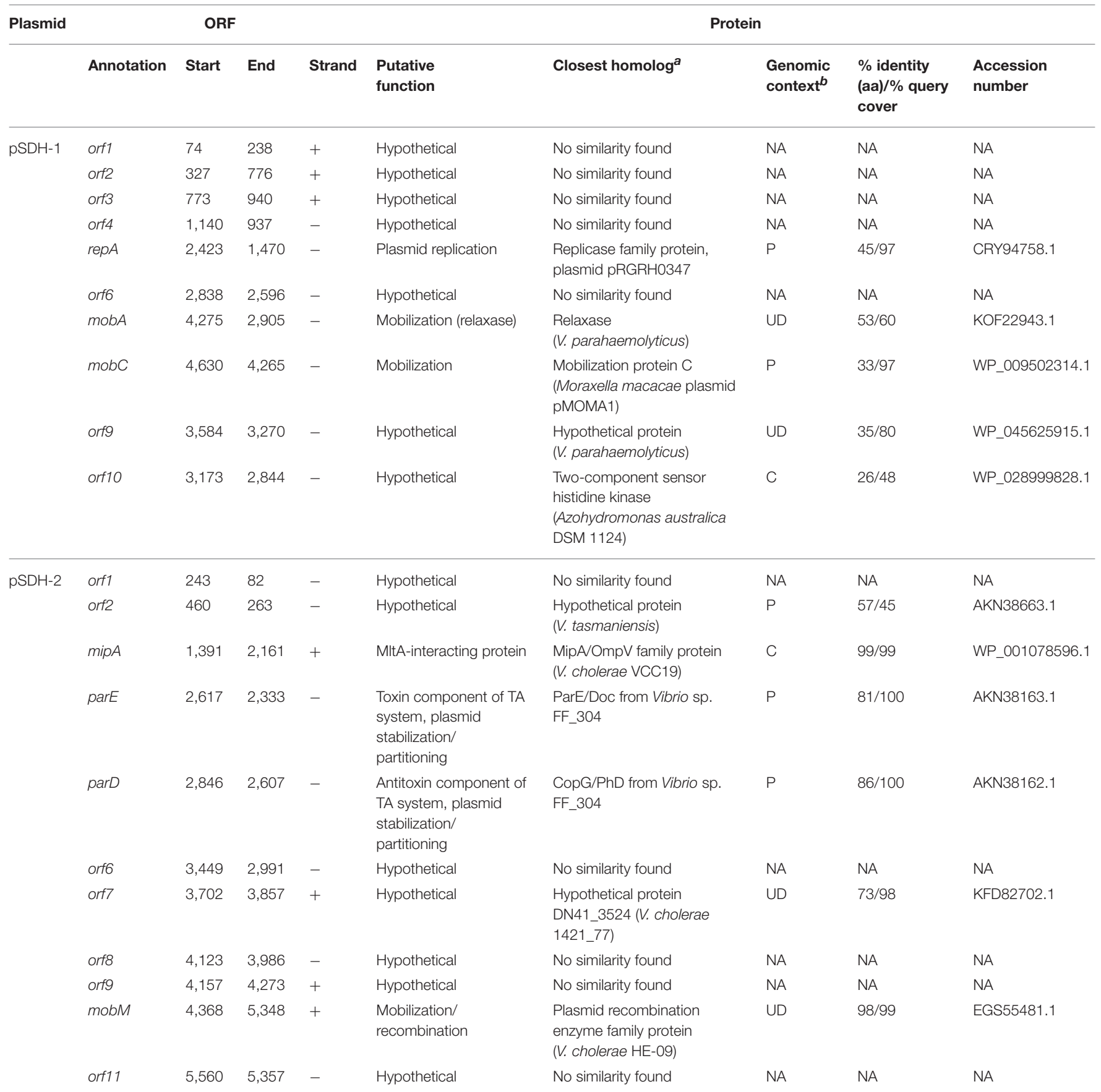

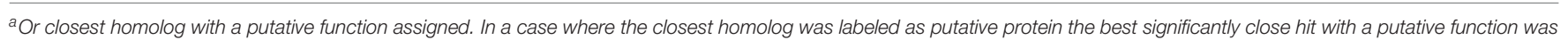
used.

${ }^{b}$ Genomic context of the homolog. P, plasmid (or extrachromosomal element); C, chromosome, UD, undetermined.

NA: not applicable

gene located directly upstream of $m o b A$ and one or two other proteins encoded by genes located within the mobA coding sequence (Francia et al., 2004). In the case of pSDH-1, we were able to identify a MobC-like protein, encoded by the ORF located directly upstream of mobA. However, the two predicted proteins encoded by the ORFs embedded in the mobA coding sequence (orf9 and orf10) do not contain any mobilization domains or features. Further analysis of the nucleotide sequence of pSDH-1 revealed a region that is similar to the origin of transfer (oriT) found in plasmids carrying a $\mathrm{MOB}_{\mathrm{HEN}}$ family relaxase (Figure 2). This region, located $52 \mathrm{bp}$ upstream the MobC translation start site contains a sequence (CTGGCTTA) that is identical to the 


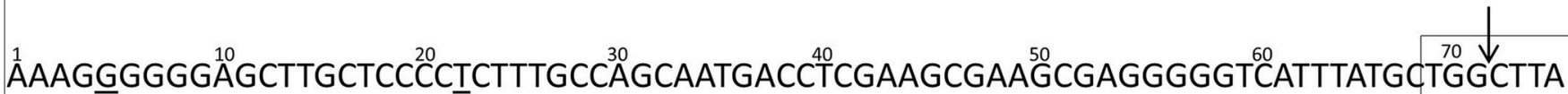

FIGURE 2 | Putative oriT region of pSDH-1. Near-perfect inverted repeats are indicated by arrows. Nucleotide mismatches are underlined. Nucleotides identical to the putative oriT region of ColE1 are boxed and the specific dinucleotide where the relaxase-mediated cleavage occurs (nic) in ColE1 is indicated by the arrow.

one found in the ColE1 oriT and which contains the cleavage site (or nic site) of ColE1 relaxase. Additionally, the putative oriT of pSDH-1 comprises two nearly perfect inverted repeats. Although the sequence of these inverted repeats differs between pSDH-1 and ColE1, they are located at a similar relative distance from the putative nic sites.

\section{Identification and Genomic Structure of plasmid pSDH-2}

Plasmid pSDH-2 encodes 11 putative ORFs (Figure 1 and Table 1). orf1, orf6, orf8, orf9, and orf11 encode putative proteins of unknown function and do not have any identifiable homologs. orf 2 encodes a putative protein that shares $57 \%$ identity on $45 \%$ of its sequence with a hypothetical protein from a Vibrio tasmaniensis plasmid. orf3 encodes a protein sharing $99 \%$ identity with a membrane protein from $V$. cholerae strain VCC19. Further analysis shows that it contains MipA/OmpV domains as well as a predicted signal peptide (Blastp and SMART). MipA (MltA-interacting protein) is believed to be a scaffolding protein for murein synthesis that mediates assembly of the lytic transglycosylase MltA with the bifunctional transglycosylase/transpeptidase $\mathrm{PBP} 1 \mathrm{~B}$, which respectively play a role in synthesis and degradation of peptidoglycan (Vollmer et al., 1999). Furthermore, UVC- and $\gamma$ - irradiation, as well as starvation, were shown to induce expression of MipA in some strains of $V$. alginolyticus (Ben Abdallah et al., 2010; Abdallah et al., 2012) suggesting a possible role in host cell persistence in the environment. pSDH-2 orf 3 was renamed mipA.

orf4 and orf5 encode a putative ParD-ParE type II toxinantitoxin (TA) system. Homologs of both these proteins were found as part of the same TA system on an extrachromosomal element, likely a plasmid, from Vibrio sp. FF_304. Type II TA systems act as plasmid stabilization systems. Loss of the plasmid by segregation results in death of the plasmid-free cell, since the toxin is more stable than its cognate antitoxin, a mechanism termed post-segregational killing. BlastP and SMART analysis revealed that the protein encoded by orf 4 contains a parE_toxin domain. A BlastP search showed that the product of orf5 contains a predicted transcriptional regulator COG3905 domain and SMART analyses further revealed that the N-terminal portion of orf5 product carries an RHH_1 motif. This ribbon-helix-helix motif is found in the ParD antitoxin of the RP4 ParD-ParE TA system, as well as in many transcriptional repressors, and is believed to mediate the DNA-binding function of ParD (Oberer et al., 2007). The unstructured C-terminal region is believed to be involved in interaction with its cognate toxin, ParE. orf4 and orf5 were respectively renamed parE and parD. orf10, renamed mobM, encodes a protein that shares $98 \%$ identity with a plasmid recombination protein from V. cholerae HE-09 and carries a SMC_proK_B domain (structural maintenance of chromosomes) at its C-terminal end. The $\mathrm{N}$-terminal region of $\mathrm{MobM}_{\mathrm{pSDH}-2}$ contains most of the conserved residues found in motifs I, II and III of Mob and Pre proteins of the pMV158 family (Francia et al., 2004). Plasmid recombination proteins such as Pre from plasmid pMV158 (also termed MobM) are relaxases involved in conjugative transfer (Priebe and Lacks, 1989; Francia et al., 2004). BlastP analysis of $\mathrm{MobM}_{\mathrm{pMV} 158}$ reveals that it also carries a C-terminal SMC_prok_B domain. Plasmids carrying a relaxase belonging to the pMV158 superfamily have been shown to be mobilizable by a large number of conjugative plasmids and integrating conjugative elements (also known as conjugative transposons) (Francia et al., 2004) (and references therein).

\section{Identification of the Putative Origin of Replication of pSDH-2}

Comparison of the nucleotide sequence of $\mathrm{pSDH}-2$ with publicly available sequences reveals that it shares no similarity with known plasmids, with the exception of two ca. $500 \mathrm{bp}$ stretches, the first located between orf 2 and mipA and the second encompassing orf7 and half of orf8. The first of these regions shares $88 \%$ similarity with an intergenic region of a mobilizable plasmid identified by in silico analysis in Vibrio tasmaniensis ZF-76 clone 102 (KP795524.1) (Xue et al., 2015). The second region shares 97\% homology with the replication region of small Vibrionaceae plasmids (Figure 3) shown to utilize a ColE1-like replication mechanism (Pan et al., 2010). Like ColE1, these CLV (ColE1like Vibrionaceae) plasmids encode two constitutively expressed and convergently transcribed RNAs (RNA I and RNA II), which respectively specify their incompatibility and replication initiation determinants (Pan et al., 2010). Studies (Pan et al., 2010; Brantl, 2014; Lilly and Camps, 2015) (and references therein) of the replication of plasmid ColE1 have shown that RNA II is the only plasmid-encoded feature required for initiation of replication. The $\sim 550$-bp pre-primer form of RNA II forms a persistent hybrid (R-loop) with the lagging-strand at the site of the origin of replication. Once the R-loop is formed, RNA II is processed by RNase $\mathrm{H}$, producing a $3^{\prime}-\mathrm{OH}$ end that serves as a primer for leading-strand synthesis. RNA I (108bp) specifies incompatibility and controls copy number of ColE1 by binding with the RNA II pre-primer and inhibiting R-loop formation. The corresponding region of $\mathrm{pSDH}-2$ contains all the features typically found in CLV plasmids, suggesting it replicates following the ColE1 model of plasmid replication (Figure 3). 


\section{A Promoter region}

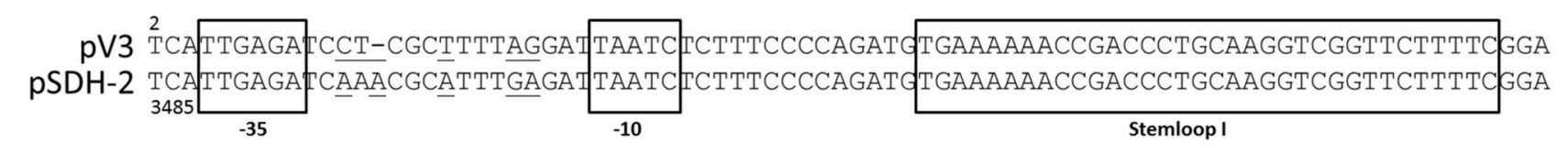

pV3 ATACTTACTAGTTTCEGC-CACTGGGTAAGTCCTTTdATGATA GCTCATATCGTTTAAGCA CTTCAAGTATTCCTGCACTCTG

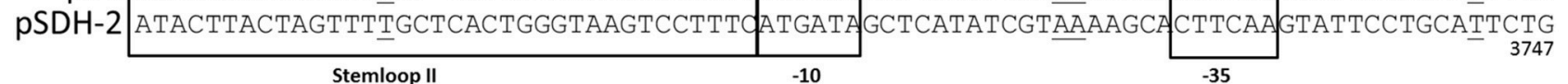

\section{B Replication region}

pV3 CGGCAGGGGTAGGGGGPCGGCGCAGCCGAAAATAAGCATTTT_CGGCGC
FSDH-2 CGGCAGGGGTAGGGGGPCGCGCAGCCGAAA-TAAGCAAATTGCGGCGC
et al., 2010). The main features of the CLV origin are indicated by boxes and their function is indicated. Nucleotides that differ between the two sequences are
underlined. The RNase H cleavage site is indicated by a white triangle. Positions on the nucleotide sequences of pSDH-2 and pV3 are indicated below and above the
alignment, respectively.

Furthermore, the regions of $\mathrm{pSDH}-2$, corresponding to the two ColE1 RNAs, are predicted to fold into stem loop structures (Figure S1).

\section{pSDH-1 and pSDH-2 Distribution in Haitian Isolates}

Two sets of primers specific for each plasmid (Figure 1) were designed to allow screening of a collection of Haitian strains, including $V$. cholerae $\mathrm{O} 1$ and $V$. cholerae non-O1/non-O139 isolated from clinical and environmental samples, and Vibrio spp. and Aeromonas spp. isolated from the same biological samples. pSDH-1 and/or pSDH-2 were predominantly detected in $V$. cholerae non-O1/non-O139 of clinical origin (34 out of 120). However, both plasmids were detected in a $V$. cholerae non-O1/non-O139 environmental isolate from the Cange district (Table 2). Two clinical isolates of $V$. cholerae $\mathrm{O} 1$ isolated in Cite Soleil also carried pSDH-1 and/or pSDH-2. 107 isolates did not hold any of the two plasmids. Other Vibrio spp. and Aeromonas spp. were all negative (data not shown). Both plasmids were geographically widespread in 8 of 13 analyzed districts, with variable prevalence in each Department (Figure 4): $33.7 \%(n=30)$ in Ouest, $14.3 \%(n=4)$ in Artibonite, $4.7 \%$ $(n=1)$ in Centre, and none in Nord Ouest (Table 2). pSDH2 was the most common element either alone $(n=14)$ or in association with pSDH-1 $(n=21)$. The latter was never detected alone. Presence of the ParD-ParE TA system on pSDH-2 may explain why this plasmid was found more frequently since the TA system would ensure maintenance. Alternatively, pSDH-2 could be mobilized at a higher frequency or by a broader range of helper conjugative elements than $\mathrm{PSDH}-1$.
TABLE 2 | Distribution of pSDH-1 and pSDH-2 among clinical $V$. cholerae non-O1/non-O139 and $\mathrm{O} 1$ isolated in different Haitian Departments.

\begin{tabular}{|c|c|c|c|c|c|c|}
\hline \multirow{2}{*}{$\begin{array}{l}\text { Department } \\
\text { (district) }\end{array}$} & \multicolumn{3}{|c|}{ V. cholerae non-01/non-0139 } & \multicolumn{3}{|c|}{ V. cholerae 01} \\
\hline & $\begin{array}{c}\text { pSDH-1 and } \\
\text { pSDH-2 }\end{array}$ & pSDH-2 ${ }^{a}$ & NP & $\begin{array}{c}\text { pSDH-1 and } \\
\text { pSDH-2 }\end{array}$ & $\mathrm{pSDH}-2^{a}$ & NP \\
\hline \multicolumn{7}{|l|}{ Artibonite } \\
\hline Dessalines & & & & & & 2 \\
\hline Gonaïves & & & 4 & & & 5 \\
\hline Saint Marc & 4 & & 3 & & & 10 \\
\hline Centre (Cange) & & $1^{*}$ & $21^{\star}$ & & & \\
\hline $\begin{array}{l}\text { Nord Ouest } \\
\text { (Bassin Bleu) }\end{array}$ & & & 1 & & & 2 \\
\hline \multicolumn{7}{|l|}{ Ouest } \\
\hline Arcahaie & & 2 & & & & 4 \\
\hline Cite Soleil & & & & 1 & 1 & 6 \\
\hline Croix-des & 3 & 2 & & & & 5 \\
\hline \multicolumn{7}{|l|}{-Bouquets } \\
\hline Delmas & 4 & 2 & 3 & & & 1 \\
\hline En Plein & & & & & & 2 \\
\hline Montrouis & & & & & & 4 \\
\hline Port-au- & 8 & 6 & 5 & & & 25 \\
\hline \multicolumn{7}{|l|}{ Prince } \\
\hline Tabarre & 1 & & 2 & & & 2 \\
\hline Total (142) & 20 & 13 & 39 & 1 & 1 & 68 \\
\hline
\end{tabular}

${ }^{a}$ Detection of only pSDH-2.

NP: no plasmid detected

* $V$. cholerae non-01/non-0139 isolates from Cange were of environmental origin. 


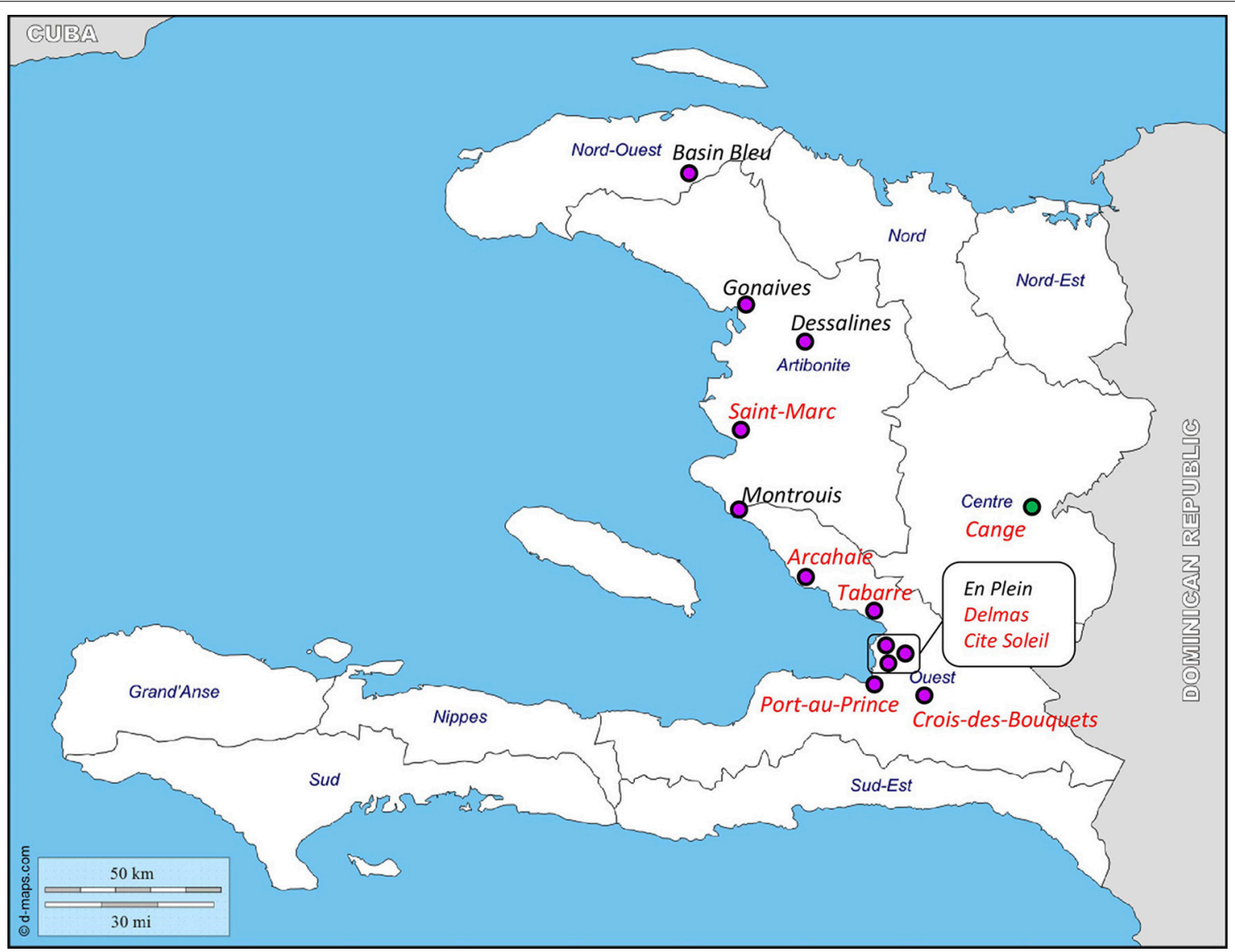

FIGURE 4 | Distribution of pSDH-1 and pSDH-2 in Haiti. Purple and green circles indicate sites where clinical and environmental isolates were collected, respectively, in the Departments of Nord-Ouest, Artibonite, Ouest, and Centre. Red indicates districts where pSDH-1 and/or pSDH-2 were detected (see Table 2). Original figure was downloaded from http://www.d-maps.com/carte.php?num_car=5262\&lang=en, according to the website terms and conditions of use.

\section{CONCLUSION}

In this study, we report two previously unknown plasmids prevalent in $V$. cholerae in the early phase of the 2010 Haitian cholera outbreak. The genome content of these plasmids suggests self-mobilization and, at least in the case of one of them, a TA system for plasmid stabilization through post-segregational killing. pSDH-1 and pSDH-2 enrich the list of small cryptic plasmids circulating in Vibrio spp. in the aquatic (Bidinost et al., 1999; Powers et al., 2000; Zhang et al., 2007) and clinical environments and whose role in bacterial fitness or pathogenicity merits further investigation.

\section{AUTHOR CONTRIBUTIONS}

Data analysis: DC, GG, and NH. Strain screening: DC. Singlecell genomics: RS. Plasmid assembly and annotation: GG, SC, and RS. Contributed materials and reviewed the paper:
MP, AH, and RC. Manuscript writing: DC and GG. All authors discussed, read, contributed to, and approved the final manuscript.

\section{FUNDING}

This research was supported by the National Institute of Allergy and Infectious Disease (NIAID) grant no. 2RO1A1039129-11A2 from the National Institutes of Health (NIH) to RC and by the National Science Foundation grant no. OCE-1335810 to RS. The funders had no role in study design, data collection and analysis, decision to publish, or preparation of the manuscript.

\section{SUPPLEMENTARY MATERIAL}

The Supplementary Material for this article can be found online at: https://www.frontiersin.org/articles/10.3389/fmicb. 2017.02283/full\#supplementary-material 


\section{REFERENCES}

Abdallah, F. B., Lagha, R., Ellafi, A., Namane, A., Rousselle, J. C., Lenormand, P., et al. (2012). Identification of outer membrane proteins altered in response to UVC-radiation in Vibrio parahaemolyticus and Vibrio alginolyticus. Indian J. Microbiol. 52, 660-665. doi: 10.1007/s12088-0120299-2

Aedo, S., Ivanova, L., Tomova, A., and Cabello, F. C. (2014). Plasmid-related quinolone resistance determinants in epidemic Vibrio parahaemolyticus, uropathogenic Escherichia coli, and marine bacteria from an aquaculture area in Chile. Microb. Ecol. 68, 324-328. doi: 10.1007/s00248-0140409-2

Ausubel, F. M., Brent, R., Kingston, R. E., Moore, D. D., Seidman, J. G., Smith, J. A., et al. (1990). Current Protocols in Molecular Biology. New York, NY: Green Publishing Associates and Wiley.

Baron, S., Lesne, J., Jouy, E., Larvor, E., Kempf, I., Boncy, J., et al. (2016). Antimicrobial susceptibility of autochthonous aquatic Vibrio cholerae in Haiti. Front. Microbiol. 7:1671. doi: 10.3389/fmicb.2016.01671

Ben Abdallah, F., Ellafi, A., Lagha, R., Bakhrouf, A., Namane, A., Rousselle, J. C., et al. (2010). Identification of outer membrane proteins of Vibrio parahaemolyticus and Vibrio alginolyticus altered in response to gamma-irradiation or long-term starvation. Res. Microbiol. 161, 869-875. doi: 10.1016/j.resmic.2010.10.009

Bidinost, C., Wilderman, P. J., Dorsey, C. W., and Actis, L. A. (1999). Analysis of the replication elements of the pMJ101 plasmid from the fish pathogen Vibrio ordalii. Plasmid 42, 20-30. doi: 10.1006/plas.1999.1406

Brantl, S. (2014). Plasmid replication control by antisense RNAs. Microbiol. Spectr. 2:PLAS-0001-2013. doi: 10.1128/microbiolspec.PLAS0001-2013

Carraro, N., Rivard, N., Burrus, V., and Ceccarelli, D. (2017). Mobilizable genomic islands, different strategies for the dissemination of multidrug resistance and other adaptive traits. Mob. Genet. Elements 7, 1-6. doi: 10.1080/2159256X.2017.1304193

Carraro, N., Rivard, N., Ceccarelli, D., Colwell, R., and Burrus, V. (2016). IncA/C conjugative plasmids mobilize a new family of multidrug resistance islands in clinical Vibrio cholerae non-O1/non-O139 from Haiti. MBio 7, e00509-00516. doi: 10.1128/mBio.00509-16

Ceccarelli, D., Spagnoletti, M., Cappuccinelli, P., Burrus, V., and Colombo, M. M. (2011). Origin of Vibrio cholerae in Haiti. Lancet Infect. Dis. 11:260. doi: 10.1016/S1473-3099(11)70078-0

Ceccarelli, D., Spagnoletti, M., Hasan, N. A., Lansing, S., Huq, A., and Colwell, R. R. (2013). A new integrative conjugative element detected in Haitian isolates of Vibrio cholerae non-O1/non-O139. Res. Microbiol. 164, 891-893. doi: 10.1016/j.resmic.2013.08.004

Chin, C.-S., Sorenson, J., Harris, J. B., Robins, W. P., Charles, R. C., Jean-Charles, R. R., et al. (2011). The origin of the haitian cholera outbreak strain. New Eng. J. Med. 364, 33-42. doi: 10.1056/NEJMoa1012928

Chomvarin, C., Jumroenjit, W., Tangkanakul, W., Hasan, N. A., Chaicumpar, K., Faksri, K., et al. (2014). Genotype and drug resistance of clinical and environmental Vibrio cholerae non-O1/non-O139 in Northeastern Thailand. Southeast Asian J. Trop. Med. Public Health 45, 1354-1364.

Crowe, S. J., Newton, A. E., Gould, L. H., Parsons, M. B., Stroika, S., Bopp, C. A., et al. (2016). Vibriosis, not cholera: toxigenic Vibrio cholerae non-O1, non-O139 infections in the United States, 1984-2014. Epidemiol. Infect. 144, 3335-3341. doi: 10.1017/S0950268816001783

Daccord, A., Ceccarelli, D., and Burrus, V. (2010). Integrating conjugative elements of the SXT/R391 family trigger the excision and drive the mobilization of a new class of Vibrio genomic islands. Mol. Microbiol. 78, 576-588. doi: $10.1111 / j .1365-2958.2010 .07364 . x$

Folster, J. P., Katz, L., McCullough, A., Parsons, M., Knipe, K., Sammons, S., et al. (2014). Multidrug-resistant IncA/C plasmid in Vibrio cholerae from Haiti Emerg. Infect. Dis. 20, 1951-1953. doi: 10.3201/eid2011.1 40889

Francia, M. V., Varsaki, A., Garcillán-Barcia, M. P., Latorre, A., Drainas, C., and de la Cruz, F. (2004). A classification scheme for mobilization regions of bacterial plasmids. FEMS Microbiol. Rev. 28, 79-100. doi: 10.1016/j.femsre.2003. 09.001
Hasan, N. A., Choi, S. Y., Eppinger, M., Clark, P. W., Chen, A., Alam, M., et al. (2012). Genomic diversity of 2010 Haitian cholera outbreak strains. Proc. Natl. Acad. Sci. U.S.A. 109, 2010-2017. doi: 10.1073/pnas.1207 359109

Hazen, T. H., Wu, D., Eisen, J. A., and Sobecky, P. A. (2007). Sequence characterization and comparative analysis of three plasmids isolated from environmental Vibrio spp. Appl. Environ. Microbiol. 73, 7703-7710. doi: 10.1128/AEM.01577-07

Hirk, S., Huhulescu, S., Allerberger, F., Lepuschitz, S., Rehak, S., Weil, S., et al. (2016). Necrotizing fasciitis due to Vibrio cholerae non-O1/non-O139 after exposure to Austrian bathing sites. Wien. Klin. Wochenschr. 128, 141-145. doi: 10.1007/s00508-015-0944-y

Hoshino, K., Yamasaki, S., Mukhopadhyay, A. K., Chakraborty, S., Basu, A., Bhattacharya, S. K., et al. (1998). Development and evaluation of a multiplex PCR assay for rapid detection of toxigenic Vibrio cholerae O1 and O139. FEMS Immunol. Med. Microbiol. 20, 201-207. doi: 10.1111/j.1574-695X.1998.tb01128.x

Letunic, I., Doerks, T., and Bork, P. (2015). SMART: recent updates, new developments and status in 2015. Nucleic Acids Res. 43, D257-D260. doi: $10.1093 /$ nar/gku949

Lilly, J., and Camps, M. (2015). Mechanisms of theta plasmid replication. Microbiol. Spectr. 3:PLAS-0029-2014. doi: 10.1128/microbiolspec.PLAS-00292014

Liu, J., Winstead-Derlega, C., Houpt, E., Heidkamp, R., Pape, J., and Dillingham, R. (2014). Pre-earthquake non-epidemic Vibrio cholerae in Haiti. J. Infect. Dev. Ctries. 8, 120-122. doi: 10.3855/jidc.4524

Oberer, M., Zangger, K., Gruber, K., and Keller, W. (2007). The solution structure of ParD, the antidote of the ParDE toxin antitoxin module, provides the structural basis for DNA and toxin binding. Protein Sci. 16, 1676-1688. doi: $10.1110 /$ ps.062680707

Pan, L., Leung, P. C., and Gu, J. D. (2010). A new ColE1-like plasmid group revealed by comparative analysis of the replication proficient fragments of Vibrionaceae plasmids. J. Microbiol. Biotechnol. 20, 1163-1178. doi: 10.4014/jmb.1003.03007

Powers, L. G., Mallonee, J. T., and Sobecky, P. A. (2000). Complete nucleotide sequence of a cryptic plasmid from the marine bacterium Vibrio splendidus and identification of open reading frames. Plasmid 43, 99-102. doi: 10.1006/plas.1999.1438

Priebe, S. D., and Lacks, S. A. (1989). Region of the streptococcal plasmid pMV158 required for conjugative mobilization. J. Bacteriol. 171, 4778-4784. doi: $10.1128 / \mathrm{jb}$.171.9.4778-4784.1989

Schultz, J., Milpetz, F., Bork, P., and Ponting, C. P. (1998). SMART, a simple modular architecture research tool: identification of signaling domains. Proc. Natl. Acad. Sci. U.S.A. 95, 5857-5864. doi: 10.1073/pnas.95. 11.5857

Stepanauskas, R. (2012). Single cell genomics: an individual look at microbes. Curr. Opin. Microbiol. 15, 613-620. doi: 10.1016/j.mib.2012.09.001

Swan, B. K., Tupper, B., Sczyrba, A., Lauro, F. M., Martinez-Garcia, M., Gonzalez, J. M., et al. (2013). Prevalent genome streamlining and latitudinal divergence of planktonic bacteria in the surface ocean. Proc. Natl. Acad. Sci. U.S.A. 110, 11463-11468. doi: 10.1073/pnas.1304246110

Vollmer, W., von Rechenberg, M., and Höltje, J. V. (1999). Demonstration of molecular interactions between the murein polymerase PBP1B, the lytic transglycosylase MltA, and the scaffolding protein MipA of Escherichia coli. J. Biol. Chem. 274, 6726-6734. doi: 10.1074/jbc.274.10.6726

Wang, P., Wen, Z., Li, B., Zeng, Z., and Wang, X. (2016). Complete genome sequence of Vibrio alginolyticus ATCC 33787(T) isolated from seawater with three native megaplasmids. Mar. Genomics 28, 45-47. doi: 10.1016/j.margen.2016.05.003

Whitehouse, C. A., Ladner, J. T., and Palacios, G. F. (2015). Molecular characterization of plasmid pMomalof Moraxella macacae, a newly described bacterial pathogen of macaques. Folia Microbiol. 60, 235-239. doi: 10.1007/s12223-014-0364-9

World Health Organization (2010). Outbreak news. Cholera, Haiti, cholera, Pakistan, Crimean-Congo haemorrhagic fever (CCHF) and dengue fever, Pakistan. Wkly. Epidemiol. Rec. 85, 437-439.

World Health Organization (2017). Weekly Epidemiological Record: Cholera. Geneva: World Health Organization. 
Xue, H., Cordero, O. X., Camas, F. M., Trimble, W., Meyer, F., Guglielmini, J., et al. (2015). Eco-evolutionary dynamics of episomes among ecologically cohesive bacterial populations. MBio 6:e00552-15. doi: 10.1128/mBio.00 $552-15$

Zhang, R., Pan, L., Zhao, Z., and Gu, J.-D. (2012). High incidence of plasmids in marine Vibrio species isolated from Mai Po Nature Reserve of Hong Kong. Ecotoxicology 21, 1661-1668. doi: 10.1007/s10646-012-0939-7

Zhang, R., Wang, Y., Leung, P. C., and Gu, J. D. (2007). pVC, a small cryptic plasmid from the environmental isolate of Vibrio cholerae MP-1. J. Microbiol. $45,193-198$.

Zuker, M. (2003). Mfold web server for nucleic acid folding and hybridization prediction. Nucleic Acids Res. 31, 3406-3415. doi: 10.1093/nar/gkg595
Conflict of Interest Statement: SC, NH, and RC were employed by company CosmosID Inc. The other authors declare that the research was conducted in the absence of any commercial or financial relationships that could be construed as a potential conflict of interest.

Copyright (๑ 2017 Ceccarelli, Garriss, Choi, Hasan, Stepanauskas, Pop, Huq and Colwell. This is an open-access article distributed under the terms of the Creative Commons Attribution License (CC BY). The use, distribution or reproduction in other forums is permitted, provided the original author(s) or licensor are credited and that the original publication in this journal is cited, in accordance with accepted academic practice. No use, distribution or reproduction is permitted which does not comply with these terms. 